\title{
Ergebnisbericht zur religionspsychologischen Studie "Intersubjektivität von Tranceerleben durch religiös-rituelle Körperhaltungen«
}

\section{Daniel Böttger}

\section{(2) OpenEdition}

\section{Journals}

Electronic version

URL: http://journals.openedition.org/zjr/720

DOI: $10.4000 /$ zjr.720

ISSN: 1862-5886

Publisher

Deutsche Vereinigung für Religionswissenschaft

\section{Electronic reference}

Daniel Böttger, « Ergebnisbericht zur religionspsychologischen Studie »Intersubjektivität von Tranceerleben durch religiös-rituelle Körperhaltungen« », Zeitschrift für junge Religionswissenschaft [Online], 3 | 2008, Online erschienen am: 31 Dezember 2008, abgerufen am 07 Mai 2019. URL : http:// journals.openedition.org/zjr/720 ; DOI : 10.4000/zjr.720

This text was automatically generated on 7 mai 2019

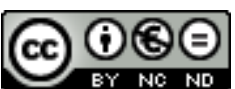

Dieses Werk ist lizenziert unter einer Creative Commons Namensnennung - Nicht-kommerziell - Keine Bearbeitung 3.0 Deutschland Lizenz. 


\title{
Ergebnisbericht zur religionspsychologischen Studie »Intersubjektivität von Tranceerleben durch religiös- rituelle Körperhaltungen«
}

\author{
Daniel Böttger
}

\section{Theorie und Fragestellung}

1 Dieser Artikel beschreibt ein Experiment, das an der Universität Leipzig im Januar 2006 durch den Autor durchgeführt wurde. Die Studie befasste sich mit der Hypothese des Vorhandenseins spezifischer "religiös-ritueller Körperhaltungen«, die unter diesem Namen durch Felicitas Goodman vorgestellt worden sind. Es handelt sich um eine Theorie älteren Datums, die vornehmlich in der Ethnologie rezipiert worden ist und den Ruf Goodmans als eine der wichtigsten Expertinnen auf dem Gebiet veränderter Bewusstseinszustände mitbegründete. Goodman wird vom religionspsychologischen Mainstream wenig rezipiert ${ }^{1}$ und Bezüge auf sie in der ethnologischen Literatur scheinen größtenteils älteren Datums zu sein. Diesem Umstand ist es offenbar geschuldet, dass eine unabhängige empirische Untersu- chung ihrer Theorie bisher nicht vorzuliegen scheint.

Die folgende inhaltliche Einführung in den Theoriekontext ist entnommen aus Andrea Baldemair (2001):Trancezendenz. Trancezustände beim Tanzen auf Tech- noparties und durch das Praktizieren religiös-ritueller Körperhaltungen nach Felicitas Goodman. ${ }^{2}$

"Seit nahezu 30 Jahren beschäftigt sich die Linguistin und Kulturanthropologin Felicitas Goodman mit der Wiederentdeckung der von ihr als >religiös-rituelk bezeichneten Körperhaltungen, einem verschütteten Kulturgut in Form einer speziellen Trance-Technik. Die Körperhaltungen, deren ursprünglich religiöse Bedeutung und tranceinduzierende Wirkung sich zufällig herausstellte, entdeckte Goodman anhand von Höhlenzeichnungen oder figurativen Darstellungen in der 
primitiven Kunst. Der Ablauf einer religiös-rituellen Trance-Sitzung wird durch einen in sich geschlossenen Handlungsablauf in Anlehnung an religiöse Zeremonien der Navajo-Indianer nachkonstruiert, zu dem in der Regel Salbeiräucherungen, das Verstreuen von Dinkelmehl sowie das Einladen und Verabschieden der Geister aus der sogenannten sanderen Wirklichkeit` gehören. Diese sandere` Wirklichkeit, welche der hiesigen Welt landschaftlich gleicht, stellt für Goodman eine ursprüngliche, religiös besetzte Dimension dar. Den Weg dorthin beschreitet man über die religiös-rituelle Trance, welche neben körperlichen Begleiterscheinungen wie Schauergefühlen, Wärmeempfindungen, Schwitzen, Zittern und einem sparadoxen Erregungszustand Eindrücken, lebhaften Begegnungen oder gar Identifizierungen mit den dort ansässigen Pflanzen- und Tiergeistern führt. Nimmt man nun eine der mittlerweile mehr als 45 erforschten Körperhaltungen über einen Zeitraum von 15 Minuten lang unter zusätzlicher Stimulation durch einen rhythmischen Klangkörper ein (Zierkürbisrassel oder Trommel; die rhythmische Empfehlung liegt bei 200 bis 250 bpm [d.h. Schlägen pro Minute, D.B.]),so entsteht dabei ein Trancezustand, der weitgehend als angenehm und positiv erlebt wird. Erstaunlich ist der Umstand, dass es - abgesehen von ernsthaften körperlichen Gebrechen und psychischen Behinderungen - weder bestimmter körperlicher noch weltanschaulicher Voraussetzungen bedarf, um Zugang zu dieser Form von Trance zu erhalten.»

Dass diese Haltungen prinzipiell in der Lage zu sein scheinen, Trancezustände hervorzurufen, konnte bereits gezeigt werden (Guttmann/Langer 1992). Erstaunlicher sind die genauen Eigenschaften dieser speziellen Trance. Der Text fährt wie folgt fort:

"Physiologische Laboruntersuchungen (vgl. Guttmann/Langer 1992) haben auf beeindruckende Weise gezeigt, dass sich bei der religiös-rituellen Trance der Gehalt an Adrenalin, Noradrenalin und Cortisol im Blutserum verringert und zugleich der Blutdruck bei gleichzeitig erhöhter Pulsfrequenz sinkt. Dieser aus medizinischer Perspektive sparadoxe Erregungszustand (paradox arousal) tritt normalerweise nur in lebensbedrohlichen Situationen auf, beispielsweise im Schockzustand oder beim Verbluten. Parallel dazu kommt es im Gehirn zur Produktion von Thetawellen und zur Ausschüttung von Beta-Endorphin. Dieses körpereigene Opiat wirkt einerseits schmerzstillend (vgl. Zehentbauer 1992), kann aber auch überwältigende Euphorie-Gefühle erzeugen, vergleichbar mit jener `Süße` religiösen Erlebens, dessen Intensität sich in Aufzeichnungen zahlreicher MystikerInnen niederschlägt (vgl. Hoffmann 1994). Insgesamt betrachtet ergibt sich bei der religiös-rituellen Trance >das Bild eines umwälzenden körperlichen Geschehens, welches beim religiösen Erlebnis die biologische Grundlage darstellt und mit dem visionären Erleben korreliert` (Goodman 1992, S. 23). Aus den Goodmanschen ErlebnisProtokollen geht zudem hervor, dass die ProbandInnen trotz unterschiedlicher kultureller Herkunft und ohne entsprechendes Vorwissen eindrucksvolle - jeweils haltungsbezogene - Übereinstimmungen im visuellen Erleben aufwiesen, sdie nicht von der Kultur des Betreffenden herstammen, sondern offensichtlich von der Haltung herrühren« (ebd. 31f).

4 Zielsetzung der hier vorgestellten Studie war es, die Behauptung im letzten Satz zu überprüfen: Haben Personen, die dieselbe Körperhaltung einnehmen, tatsächlich automatisch ähnliche Tranceerlebnisse? In anderen Worten: Sind die Tranceerlebnisse bei der Ausübung "rituell-religiöser Körperhaltungen« nach Felicitas Goodman intersubjektiv?

5 Die Frage liegt vor allem aus dem Grund nahe, da Goodman ihre Versuche mit den Körperhaltungen als solche in kleinen Gruppen und ohne Doppelblindheit beschreibt, bei denen die Probanden offenbar die Möglichkeit hatten, Beschreibungen anderer Probanden zu hören, bevor sie ihre eigenen Ergebnisse zu verbalisieren hatten. Eine scheinbare Ähnlichkeit der verschiedenen Tranceerlebnisse hätte durch eine solche 
unsaubere Verfahrensweise zustande kommen können. Die Überprüfung der Frage, ob diese Techniken überhaupt zu Trance- zuständen führen, war demnach ausdrücklich nicht Ziel der Studie.

\section{Versuchspersonen}

6 Die Versuchspersonen (im Folgenden Vpn) waren 61 Freiwillige, größtenteils Studenten und Studentinnen. Voraussetzungen für die Teilnahme war körperliche und geistige Gesundheit, Unkenntnis der Beschreibungen der (nach Goodman) "typischen" Tranceerlebnisse und bei weiblichen Teilnehmern Nicht-Gravidität. Die Erfüllung der Voraussetzungen versicherten die Vpn gemeinsam mit ihrer Einverständniserklärung schriftlich. Weitergehende psychologische und medizinische Daten wurden nicht erhoben, da sie nach Goodmans Darstellung für die Hypothese unerheblich wären.

\section{Versuchsablauf}

7 Der Versuch fand im Religionswissenschaftlichen Institut der Universität Leipzig am 31.1.2006 statt. Alle Teilnehmer wurden gemeinsam in einem Kurzvortrag über Goodmans Theorie informiert, etwa wie im Abschnitt Theoriekontext geschehen. Es wurden keine Beispiele für mögliche Inhalte des Tranceerlebens genannt, sondern die Vielfalt möglicher Erlebnisse für Trance-Unerfahrene mit der von Träumen verglichen. Alle Fragen zum geplanten Versuchsablauf (außer betreffend der hypothetischen Inhalte der zu erwartenden Erlebnisse) wurden beantwortet.

8 Nachdem alle Vpn der Teilnahme zugestimmt hatten, erhielten sie Schreibwerkzeuge und Bögen mit der Erklärung des Einverständnisses zur Teilnahme und der Kenntnis ihrer Rechte als Probanden. Die Vpn erhielten fort- laufende Nummern. Nachdem die Erklärungen unterschrieben und eingesammelt waren, spielten sie für den weiteren Versuchsablauf keine Rolle. Die Vpn wurden (durch einfaches Durchzählen) randomisiert in vier Gruppen von einmal 16 und dreimal 15 Mitgliedern aufgeteilt. Jede Gruppe erhielt eine Einweisung in eine von vier Haltungen. Die einweisende Yogalehrerin und Psychologiestudentin Katja Schlegel war nicht über die den Haltungen zugeordneten Tranceinhalte informiert. Diese Bedingung (Doppelblindheit) war wichtig, um auszuschließen, dass die Vpn durch unterschwellige Kommunikation Hinweise darauf erhalten würden, was sie erleben "sollten."

Alle Vpn blieben im selben Raum, mit dem Gesicht zur Wand stehend. Auf ein Zeremoniell der eingangs beschriebenen Art wurde verzichtet, um etwaige religi- öse Gefühle der Vpn nicht zu verletzen. Um dennoch in Übereinstimmung mit den gängigen ethnologischen Ritualtheorien (vgl. Belliger, Krieger 1998) einen Übergang vom Alltagsraum zum Ritualraum zu markieren, wurde eine Entspan- nungsübung (Tiefatmung) durchgeführt, die den Beginn einer Phase völligen Schweigens markierte. Außerdem wurde das elektrische Licht durch Kerzenlicht ersetzt.

Ein CD-Track mit einem Goodmans Anforderungen entsprechenden Rhythmusgeräusch von 15 Minuten Länge wurde abgespielt. ${ }^{3}$ Dabei nahmen die Vpn jeweils die ihnen zugewiesene Haltung ein. Die Haltungen lauten wie folgt (bei allen Vpn sind die Augen geschlossen): ${ }^{4}$ 
1. »Man steht aufrecht, die Füße sind parallel zueinander und etwas auseinander gestellt, die Knie werden leicht gebeugt; der rechte Arm hängt gerade nach unten, der linke Arm liegt mit gestreckter Hand auf dem Oberkörper so, daß der Unterarm einen rechten Winkel mit dem Oberarm bildet.« (S. 93)

2. »Man steht mit den Füßen parallel, mit leicht gekrümmten Knien. Beide Hände werden gekrümmt, so als würde man einen kleinen Gegenstand, etwa ein Taubenei, umklammern. Dann legt man die Hände so aneinander, daß sich der erste Knöchel des Mittelfingers berührt. Macht man es richtig, dann formen die geballten Hände ein Dreieck. Dann legt man die Hände auf den Leib, so daß der Nabel sich in diesem Dreieck befindet, wie die Dachluke unter einem spitzen Dach. Die Daumen liegen locker nebeneinander auf den Fingern. Die Oberarme stützen sich leicht am Oberkörper an. Den Kopf lehnt man zurück, so als wollte man die Kante zwischen Wand und Decke sehen.« (S. 97)

3. »Die Knie werden leicht gekrümmt, der Kopf ist etwas zurückgelegt, der Mund wird geöffnet, indem man die untere Kinnlade fallen läßt, aber man gibt keinen Laut von sich und atmet durch die Nase. Die Hände werden gespreizt in die Leistengegend gelegt.« (S. 116)

4. »Man steht aufrecht, mit den Füßen parallel und eine Spanne auseinander. Die Knie sind leicht gekrümmt, die Arme liegen auf der Brust in der Weise, daß sich der linke Arm oberhalb des rechten befindet. Der Abstand zwischen den Armen wird verschieden angegeben und scheint das Erlebnis nicht zu beeinflussen.« (S. 116)

11 Alle Vpn hatten jederzeit die Möglichkeit, abzubrechen, waren jedoch gebeten, sich in diesem Fall sehr ruhig zu verhalten und die anderen Vpn nicht zu stören. Für etwaige medizinische Notfälle befand sich der Facharzt Carsten Siegert im Nebenraum. Zwei Teilnehmer mussten mit Schwindelgefühlen abbrechen - eine Teilnehmerin stürzte dabei - und wurden ärztlich versorgt, erholten sich aber ohne weiteres schnell.

12 Aufgrund eines technischen Defektes brach die Audiowiedergabe nach nicht ganz 13 Minuten $\mathrm{ab}$ - daraufhin wurde so verfahren wie für das reguläre Ende der Audiowiedergabe geplant. Die Vpn wurden gebeten, die Haltungen zu beenden und eine abschließende Tiefatem-Übung mitzumachen. Dann erhielten alle Vpn folgende zwei Fragebögen:

Fragebogen1

Zur Studie »Intersubjektivität von Tranceerleben durch religiös-rituelle Körperhaltungen «:

Bitte beschreiben Sie den Inhalt der Gedanken, Eindrücke und Bilder, die sie hatten, als Sie die religiös-rituelle Körperhaltung eingenommen haben. Bitte gehen Sie nicht darauf ein, wie leicht oder schwer es Ihnen fiel, Tranceeindrücke zu erhalten oder was Sie über das Experiment und Ihre Umgebung dachten. Bitte schreiben Sie leserlich. Vielen Dank!

Wenn Sie mehr erlebt haben, als auf diese Zeilen passt, fassen Sie bitte nur die für Sie wichtigsten Themen und Abläufe zusammen.

Wenn Sie wenig oder so gut wie nichts erlebt haben, beschreiben Sie bitte, welche Eindrücke Sie während der 15 Minuten richtig oder passend gefunden hätten oder von was für Eindrücken Sie denken, dass Ihre Haltung sie hätte bewirken können, wenn sie funktioniert hätte.

Die Aufforderung, nötigenfalls auch nur »richtige« oder »passende« Tranceerlebnisse zu notieren, hatte den Hintergrund, dass Tranceerlebnisse teils schwer verbalisierbar sein können. Von Teilnehmern, die sich über ihre Erlebnisse nicht ausreichend im Klaren waren sollten so dennoch Daten über die Qualität der Eindrücke erhalten werden - sofern überhaupt möglich. Der sehr begrenzte Platz auf den Fragebögen und die Bitte, gegebenenfalls zusammenzufassen, dienten dazu, die Beschreibungen in ihrer Form denjenigen von Felicitas Goodman vergleichbar zu machen. Diese haben ähnliche Länge, 
siehe unten. Beide Vorgehensweisen - sowohl die Priorisierung von eventuell sehr vielen Erlebnissen, als auch die Beschreibung von nur als potentiell erlebten Eindrücken - sind prinzipiell problematisch, weil sie das unreflektierte Wiedergeben der Eindrücke einschränken. Sie schienen als Kompromiss zwischen der freien Wiedergabe und den Anforderungen der analytischen Vergleichbarkeit erforderlich, ohne den die später folgende Inhaltsanalyse so nicht hätte stattfinden können.

Um zu verhindern, dass die in Fragebogen 2 zur Auswahl stehenden Möglichkeiten die Antworten auf Fragebogen 1 beeinflussen würden, wurde Fragebogen 2 erst verteilt, als Fragebogen 1 vollständig eingesammelt worden war. Die Teilnehmer wurden daran erinnert, auch während der Beantwortung dieser Fragen nicht miteinander zu sprechen.

Fragebogen 2

Zur Studie „Intersubjektivität von Tranceerleben durch religiös-rituelle Körperhaltungen«

Bitte kreuzen Sie an, inwiefern folgende Aussagen auf die von Ihnen in Fragebogen 1 geschilderten Inhalte zutreffen. Wenn Sie wenig oder so gut wie nichts erlebt haben, beziehen Sie sich hier bitte wieder auf die passend oder möglich scheinenden Eindrücke.

\begin{tabular}{|l|l|l|l|l|}
\hline & $\begin{array}{c}\text { trifft stark } \\
\mathrm{zu}\end{array}$ & $\begin{array}{c}\text { trifft eher } \\
\mathrm{zu}\end{array}$ & $\begin{array}{l}\text { trifft eher } \\
\text { nicht zu }\end{array}$ & $\begin{array}{c}\text { trifft nicht } \\
\mathbf{z u}\end{array}$ \\
\hline $\begin{array}{l}\text { Ich hatte das Gefühl, einer } \\
\text { heilenden Kraft zu } \\
\text { begegnen, die mir helfen } \\
\text { wollte/ geholfen hat. }\end{array}$ & & & & \\
\hline $\begin{array}{l}\text { Ich habe viele verschiedene } \\
\text { schöne Dinge gesehen, wie } \\
\text { bei einem Ferienausflug. }\end{array}$ & & & & \\
\hline $\begin{array}{l}\text { Ich habe vor allem eine } \\
\text { Höhle oder Dunkelheit und } \\
\text { Tiefe erlebt. }\end{array}$ & & & & \\
\hline $\begin{array}{l}\text { Ich hatte den Eindruck, } \\
\text { nach oben zu wachsen und } \\
\text { mich zu verwandeln. }\end{array}$ & & & & \\
\hline $\begin{array}{l}\text { Ich habe mich längere Zeit } \\
\text { so gefühlt, als ob ich über } \\
\text { die Erde fliegen würde. }\end{array}$ & & & & \\
\hline $\begin{array}{l}\text { Es schien, als würden eine } \\
\text { Reihe von Tieren und } \\
\text { Vögeln zu mir kommen. }\end{array}$ & & & & \\
\hline $\begin{array}{l}\text { Ich habe ein einzelnes Tier } \\
\text { getroffen, das ein wichtiges } \\
\text { Thema meiner Erlebnisse } \\
\text { war. }\end{array}$ & & & & \\
\hline $\begin{array}{l}\text { Meine Erlebnisse schienen } \\
\text { mit dem Thema Tod zu tun } \\
\text { zu haben. }\end{array}$ & & & & \\
\hline
\end{tabular}

Die Beleuchtung wurde in ihren Normalzustand zurückversetzt. Das Schweigen wurde beendet und die Teilnehmer konnten sich entspannen und Erfrischungen zu sich nehmen. Damit war das Experiment beendet.

Die Vpn waren aber eingeladen, die Auswertung abzuwarten und noch vor Ort das Ergebnis zu erfahren. Mit der Auswertung wurde sofort begonnen. Das sollte einerseits die Neugier der Beteiligten befriedigen, andererseits aber auch weitere Aspekte der experimentellen Arbeit vorstellen ohne eigene Mitarbeit zu erfordern und konnte schließlich auch als Belohnung für die Teilnahme empfunden werden. 


\section{Auswertung}

17 Die ausgefüllten Fragebögen 1 wurden in einem abgesonderten Raum einer qualitativen Textanalyse unterzogen. Es waren die einzelnen Beschreibungen der Vpn jeweils derjenigen von Goodmans Beschreibungen zuzuordnen, in der die meisten semantischen Elemente wiederkehrten. Es wurden also Gruppen von ausgefüllten Fragebögen gebildet, die jeweils einer der Beschreibungen am meisten ähnelten Die Zuordnung wurde wiederum durch Katja Schlegel doppelblind - ohne die Information, welche von Goodmans Beschreibungen zu welcher Haltung gehört - vorgenommen. Völlig fehlende Tranceerlebnisse wurden aussortiert, da die Tranceinduktion selbst nicht Gegenstand der Untersuchung war. Die Beschreibungen zu den Körperhaltungen lauten (vgl. Goodman 1996):

1. Fahrt über die Mittlere Welt [d.h. über die Erde, D.B.] (Thema Seelenfahrt)

Die Erlebnisse erinnern an einen Bericht aus Ostasien, wo der Schamane die Totenseele noch einmal über die Erde führt, damit sie sich zum letzten Mal an der Schönheit der Heimat erfreuen kann. Die Seelenfahrt findet waagerecht zur Erde statt, die Erlebnisse sind außerordentlich vielseitig, wie bei einem Ferienausflug, und meist von großer Heiterkeit.

2. Die Haltung des Bärengeistes (Thema Heilung)

Bei dieser Haltung wird oft berichtet, daß man gespalten wird und eine Flüssigkeit eingeträufelt bekommt. Aber das Heilen kann auch viele andere Formen annehmen. Die Energie strömt z.B. aufwärts oder sammelt sich an der kranken Stelle. Man kann von der Bärenkraft geschüttelt oder gestoßen werden. Hingefallen ist aber noch niemand bei dieser Behandlung. Der Bärengeist er- scheint oft persönlich, und sein Erscheinen wird fast immer von einer leuchtend violetten Farbe begleitet. Oder es kommt einer seiner Gehilfen, die Schlange, der Dachs, der Adler oder der Berglöwe. Die heilende Kraft des mächtigen Bärengeistes wirkt vornehmlich auf den Teilnehmer/die Teilnehmerin, man kann aber auch um Heilung für Abwesende bitten. Der Bärengeist nimmt außerdem des öfteren die Gelegenheit wahr, um eine/ $\mathrm{n}$ Teilnehmer/in zu weihen, indem er ihn in Trance zerstückelt und dann wieder zusammenfügt, ohne daß das Erlebnis als leid- oder qualvoll empfunden wird.

3. Das Rufen der Geister (Thema Metamorphose)

Man verwandelt sich meist in ein hohes Gebilde, in einen Berg, eine Säule oder vor allem in einen Baum. Als solcher wiegt man sich, die Krone schwingt, man wird vom Wind bewegt. Dann kommen Tier- und Vogelgeister heran. Wir meinen, es handelt sich bei dieser Verwandlung um den Weltenbaum.

4. Der Ritt zum Totenreich (Thema Tod)

Das Vorfeld des Totenreiches erscheint dunkel und öde. Nach Ablegung des Körpers gelangt man schließlich an eine dunkle Kuhle oder einen Kessel, in dem mit Hilfe eines Tiergeistes ein neuer Körper erworben werden kann.

Währenddessen wurden die Daten aus dem zweiten Fragebogensatz digitalisiert. Nach erfolgter Zuordnung wurden auch die Ergebnisse der Textanalyse digitalisiert. Anhand der gewonnenen Daten wurde die Hypothese computergestützt überprüft. Die Ergebnisse der qualitativen Analyse waren durch einfache Zuordnungshäufigkeiten zu erfassen.

Die Fragen auf Fragebogen 2 operationalisieren Goodmans vier Beschreibungen in je zwei Items. Die einzelnen Antworten hatten Zahlenwerte: 2 für »trifft stark zu«, 1 für »trifft eher zu«, -1 für "trifft eher nicht zu«, -2 für "trifft nicht zu«. Ein genau Goodmans Vorgaben entsprechendes Erlebnis würde zu positiven Antworten auf die der Haltung entsprechenden Items und negativen Antworten auf die zu den drei anderen Haltungen 
gehörenden Items führen. Der Ähnlichkeitsindex wurde deshalb in folgender Weise berechnet.

$$
I=\frac{\left(p_{1}+p_{2}\right)}{8}-\frac{\left(n_{1}+n_{2}+n_{3}+n_{4}+n_{5}+n_{6}\right)}{24}
$$

$p$ sind dabei die Werte der zwei Items, die zu der Haltung gehören und die positiv sein müssen, um Ähnlichkeit anzuzeigen. $n$ sind die Werte der sechs konkurrierenden Items, die bei Ähnlichkeit negativ sein müssen. Der maximale Ähnlichkeitsindex I von 1 wäre ein vollständig Goodmans Beschreibungen entsprechen- des Ergebnis, eine -1 wäre ein vollkommen entgegengesetztes. Gleichmäßig verteilte Antworten - etwa ein »trifft nicht zu« auf alle 8 Fragen - führen zu einem Index von 0.

Für jeden Fragebogen wurden vier Indizes berechnet; die Ähnlichkeiten zu jeder der vier Haltungen. Anschließend wurde ausgewertet, wie ähnlich die Beschreibungen derjenigen Vpn, die Haltung 1 eingenommen hatten, Goodmans Beschreibung von Haltung 1 waren, wie sehr sie eher Haltung 2 ähnelten usw. Die Ergebnisse (Abschnitt 5) wurden allen Vpn mitgeteilt. Es folgte eine für alle Vpn offene Diskussion der Ergebnisse.

\section{Ergebnisse}

Im Fall des ersten Fragebogens zeigte sich, dass ein Großteil der Erlebnisse der Teilnehmer keiner einzigen der vorgegebenen Beschreibungen auch nur entfernt ähnelte. Die zur Verfügung stehende Zeit reichte für die Textanalyse aus - das Material war wenig umfangreich und in der Form recht homogen - aber die gesuchten semantischen Elemente waren schwer zu finden. Meist war es nötig, weit hergeholte Zuordnungen zu treffen. So etwa bei folgender Beschreibung, die nur der Haltung 2 zugeordnet werden konnte, weil das Stichwort "violett « vorkam: ${ }^{5}$

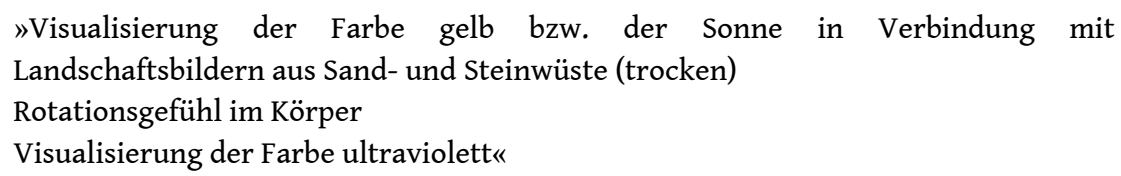

In vielen Fällen war es selbst mit einer so großzügigen Auslegung von »Übereinstimmung « nicht möglich, die Erlebnisse der Vpn zuzuordnen. Das gilt insbesondere für diejenigen Personen, bei denen sich die Trance keineswegs so automatisch einstellte wie von Goodman geschildert:

"Die Haltung hat bei mir nichts bewirkt, eher die Musik. So hatte ich anfangs das Bild eines tanzenden Naturvolkes: Doch die meiste Zeit hatte ich keinerlei Bilder. Meiner Meinung nach bewirkt die Haltung nichts als Halsschmerzen. Wenn etwas Eindrücke hervorruft dann ist es höchstens die Musik."

Die Aufforderung, nötigenfalls auch nur "richtige« oder "passende» Tranceerlebnisse zu notieren, war von den Vpn offenbar ignoriert worden. In diesen - zahlreichen - Fällen wurden die Beschreibungen aussortiert. Die Fragebögen ein- gerechnet, bei denen die Vpn ihre Nummer nicht angegeben hatten, mussten 44 aussortiert werden und es blieben nur 17, bei denen eine Zuordnung möglich war. 


\begin{tabular}{|l|l|l|l|l|l|c|}
\cline { 3 - 7 } \multicolumn{1}{c|}{} & \multicolumn{4}{c|}{ In Textanalyse zugeordnete Haltung } & Gesamt \\
\cline { 2 - 7 } \multicolumn{1}{c|}{} & 1 & 2 & 3 & 4 & \\
\hline Eingenommene & 1 & 3 & 1 & 0 & 0 & 4 \\
Haltung & 2 & 3 & 0 & 0 & 0 & 3 \\
& 3 & 2 & 1 & 1 & 2 & 6 \\
\multirow{3}{*}{ Gesamt } & 4 & 0 & 0 & 3 & 1 & 4 \\
\hline
\end{tabular}
denjenigen in den unmarkierten Feldern liegen würden. ${ }^{6}$ Das ist wiederum offensichtlich nicht der Fall. Die allein von der eingenommenen Haltung abhängigen Inhalte von Tranceerleben bei religiös-rituellen Körperhaltungen konnten nicht bestätigt werden. Stattdessen bestätigen die Ergebnisse die Nullhypothese; sie sprechen dafür, dass die Übereinstimmungen in den Ergebnissen von Felicitas Goodman und von Rezipienten/innen wie Andrea Baldemair andere Gründe haben.

\section{Diskussion}

Ein möglicher Erklärungsversuch wäre, dass die zwei aufgetretenen Störungen - die kurze Unruhe, als eine Teilnehmerin stürzte, oder die Tatsache, dass der Trommelrhythmus etwas zu früh aussetzte - die Intersubjektivität der Tranceerlebnisse verhindert haben. Goodman macht in ihrer Theorie keine Angaben über die möglichen Auswirkungen solcher Ereignisse. Ebenso wenig gibt sie beispiels- weise an, ob die vergleichsweise hohe Anzahl von 61 Personen oder die vier verschiedenen Haltungen zur 
gleichen Zeit dazu führen könnten, dass Intersubjektivität der Tranceerlebnisse nicht zu erwarten wäre. Auch eine Erklärung dafür, dass ein Großteil der Vpn keine Trancebilder erlebt hat, fehlt.

In jedem Fall ist die einfache Hypothese der Intersubjektivität ohne weitere (möglicherweise verletzte) Bedingungen nicht mit den Daten $\mathrm{zu}$ vereinbaren. Es ist naheliegend, stattdessen als mögliche andere Gründe für die bei Goodman vorhandenen Übereinstimmungen das Fehlen von Doppelblindheit und die vergleichsweise kleinen Probandenzahlen anzunehmen. Das hieße, dass Mängel in den entsprechenden Versuchsanordnungen zum Auftreten des vermeintlichen Effektes geführt hätten. An dieser Stelle kann nur gemutmaßt werden, weil Frau Goodman ihre Versuchsanordnungen nicht im Detail beschreibt.

$\mathrm{Ob}$ und welche anderen Faktoren ausschlaggebend waren, ist beim derzeitigen Stand der Forschung zur Induktion veränderter Bewusstseinszustände kaum zu er- mitteln. Es liegen zwar Studien beispielsweise zur Wirkung von Fasten, Reizentzug, Reizüberflutung, Hyperventilation, verschiedenen Drogen und anderen Methoden vor, die in verschiedenen Religionen verwendet werden, um besondere Bewusstseinszustände $\mathrm{zu}$ erreichen (vgl. Wulff 1997). Jedoch hat noch kein umfassendes Modell des Bewusstseins und seiner Zustände, wie sie auch religiöse Handlungen begleiten, breite Akzeptanz gefunden (Schuster 2005). Das führt dazu, dass teils grundverschiedene Zustände unter » Trance» oder "Meditation« zusammengefasst werden, schlicht weil es keine zulängliche Terminologie (geschweige denn eine religionspsychologisch taugliche, mehrdimensionale Definition) für sie gibt (Walsh 1990). Die Untersuchungen, die zum Thema vorliegen, befassen sich mit Vorliebe mit individuellen Erlebnissen, nicht aber mit den potentiell komplexen Zusammenhängen in einer experimentellen (und erst recht einer rituellen) Gruppensituation. ${ }^{7}$ Das ist umso erstaunlicher, da eine starke Fraktion innerhalb der Religionspsychologie diese Bewusstseinszustände (sofern sie religiös gedeutet werden) und ihre neuronalen Korrelate für die Grundlage von Religiosität überhaupt halten (Wulff 1997).

31 Der Versuch zeigt nicht zuletzt aber auch, dass experimentelle religionswissenschaftliche Forschung eine reizvolle und spannende Aufgabe ist, die sich auch Studierende zutrauen dürfen, eine passende Aufgabenstellung vorausgesetzt. Die beschriebene Studie hat weniger Arbeitsaufwand gekostet als die meisten Hausarbeiten und hat durchaus einen bescheidenen Erkenntnisgewinn erbracht. Es war vor allem durch den freundlichen Rat verschiedener Dozenten/-innen und der Hilfe einiger Kommilitonen/-innen überraschend einfach, das Experiment in Eigenverantwortung durchzuführen.

Wenn sich deshalb weitere Studierende ermutigt fühlen, die experimentelle Arbeit selbst auszuprobieren, so hat dieser Artikel - neben dem rein wissenschaftlichen - auch sein zweites Ziel erreicht. 


\section{BIBLIOGRAPHY}

BELLIGER, Andréa, KRIEGER, David J. (Hrsg.) (1998): Ritualtheorien. Opladen: Westdeutscher Verlag.

CARDEÑA, Etzel, LYNN, Steven J., KRIPPNER, Stanley C. (2000): Varieties of Anomalous Experience: Examining the Scientific Evidence. Washington DC: American Psychological Association.

DUNDE, S.R. (Hrsg.) (1993): Wörterbuch der Religionspsychologie. Gütersloh: Gütersloher Verlagshaus.

GOODMAN, Felicitas (1992, 1996): Trance - der uralte Weg zum religiösen Erleben. Gütersloh: Gütersloher Verlagshaus.

GOODMAN, Felicitas (1989): Wo die Geister auf den Winden reiten. Trancereisen und ekstatische Erlebnisse. Freiburg im Breisgau: Hermann Bauer.

GUTTMANN, G. und G. LANGER (1992): Das Bewußtsein. Multidimensionale Entwürfe. Wien, New York: Springer.

HOFFMAN, Kayne (1994):Dimensionen der Ekstase. München: Anarche.

SCHUSTER, Lars (2005):Mens ex Machina. Technische Modelle in der Bewusstseinsforschung. Frankfurt am Main, Berlin, Bern, Bruxelles, New York, Oxford, Wien: Peter Lang.

WALSH, Roger (1990):The Spirit of Shamanism. New York: Tarcher.

WULFF, David (1997):Psychology of Religion: Classic and Contemporary. New York: John Wiley \& Sons.

ZEHENTBAUER, Josef (1992):Körpereigene Drogen. Die ungenutzten Fähigkeiten unseres Gehirns. München, Zürich: Armetis und Winkler.

\section{NOTES}

1. Eine Ausnahme ist ihre Mitautorinnenschaft in (DUNDE 1993). Darin verweist sie ebenfalls auf ihre Theorie religiös-ritueller Körperhaltungen.

2. Veröffentlicht in: Beiträge zur Popularmusikforschung, Beiträge 27/28, S. 253-269.

3. Von der CD »Ecstatic Trance« von Nana NAUWALD, einer Schülerin von GOODMAN.

4. Alle Beschreibungen aus (GOODMAN 1996).

5. Diese Vpn hatte Haltung 3 eingenommen.

6. Das würde bedeuten, dass im Schnitt die Angaben der Personen, die Haltung 1 eingenommen hatten, überwiegend GOODMANs Beschreibung von Haltung 1 ähnelten, die der Personen mit Haltung 2 der Beschreibung für Haltung 2 usw.

7. Einen Überblick über die individuumsbezogene Forschung bietet CARDEÑA, LYNN, KRIPPNER 2000. 


\section{ABSTRACTS}

Der Artikel beschreibt ein Experiment, das 2006 an der Universität Leipzig durch den Autor durchgeführt wurde. Darin sollte die Hypothese überprüft werden, bestimmte von der Anthropologin Felicitas Goodman publizierte Körperhaltungen würden unter bestimmten Bedingungen $\mathrm{zu}$ stets ähnlichen Tranceerlebnissen führen. Nach einer Kurzdarstellung von Goodmans Theorie wird das Experiment vergleichsweise detailliert beschrieben. Ein Schwerpunkt liegt auf der Auswertung der Daten. Das Ergebnis widerspricht der Hypothese und wird im Abschluss kurz diskutiert.

The article describes an experiment conducted by the author at the University of Leipzig in 2006. The experiment was an attempt to test the hypothesis that certain body postures described by the anthropologist Felicitas Goodman would, under certain circumstances, always lead to similar trance experiences. After a brief outline of Goodmans theory, the experiment is described in detail with emphasis placed on the interpretation of data. The result turns out to contradict the hypothesis and is then briefly discussed.

\section{AUTHOR}

\section{DANIEL BÖTTGER}

Daniel Böttger hat von 2004 bis 2006 in Leipzig Religionswissenschaft, Psychologie und Ethnologie studiert. Derzeit studiert er Religionswissenschaft im Masterstudiengang. Kontakt:daniel.boettger@gmail.com, http://daniel.boettger.googlepages.com 